\title{
Chitin Triggers Calcium-Mediated Immune Response in the Plant Model Physcomitrella patens
}

\author{
Giulia Galotto, ${ }^{1}$ Isidro Abreu, ${ }^{2}$ Catherine Sherman, ${ }^{1,3}$ Boyuan Liu, ${ }^{1}$ Manuel Gonzalez-Guerrero, ${ }^{2,4}$ and \\ Luis Vidali',3,† \\ ${ }^{1}$ Department of Biology and Biotechnology, Worcester Polytechnic Institute, Worcester, MA, U.S.A. \\ ${ }^{2}$ Centro de Biotecnología y Genómica de Plantas (UPM-INIA), Universidad Politécnica de Madrid, Madrid, Spain \\ ${ }^{3}$ Bioinformatics and Computational Biology Program, Worcester Polytechnic Institute, Worcester, MA, U.S.A. \\ ${ }^{4}$ Department of Biotechnology-Plant Biology, Escuela Técnica Superior de Ingeniería Agronómica, Alimentaria y de \\ Biosistemas, Universidad Politécnica de Madrid, Madrid, Spain
}

Accepted 1 April 2020.

\begin{abstract}
A characteristic feature of a plant immune response is the increase of the cytosolic calcium $\left(\mathrm{Ca}^{2+}\right)$ concentration following infection, which results in the downstream activation of immune response regulators. The bryophyte Physcomitrella patens has been shown to mount an immune response when exposed to bacteria, fungi, or chitin elicitation, in a manner similar to the one observed in Arabidopsis thaliana. Nevertheless, whether the response of $P$. patens to microorganism exposure is $\mathrm{Ca}^{2+}$ mediated is currently unknown. Here, we show that $P$. patens plants treated with chitin oligosaccharides exhibit $\mathrm{Ca}^{2+}$ oscillations, and that a calcium ionophore can stimulate the expression of defense-related genes. Treatment with chitin oligosaccharides also results in an inhibition of growth, which can be explained by the depolymerization of the apical actin cytoskeleton of tip growing cells. These results suggest that chitin-triggered calcium oscillations are conserved and were likely present in the common ancestor of bryophytes and vascular plants.
\end{abstract}

Keywords: actin cytoskeleton, bryophytes immune response, calcium, chitin oligosaccharides, defense signaling pathways, Physcomitrella patens, plant responses to pathogens

In nature, both abiotic stresses (e.g., salinity, dehydration, and high UV exposure) and biotic stress (pathogen attack) are a threat to plant growth and survival. When plants are attacked by a microbial pathogen, a defense response cascades from the recognition of the pathogen to the expression of defense-related genes. In plants, pattern recognition receptors (PRR) expressed on the plasma membrane recognize pathogen-associated and microbe-associated molecular patterns (PAMPs and MAMPS,

${ }^{\dagger}$ Corresponding author: L. Vidali; lvidali@wpi.edu

Funding: Funding was provided by the European Research Council Starting Grant ERC-2013-StG-335284 to M. Gonzalez-Guerrero, by the Ministerio de Ciencia, Innovación y Universidades (FJCI-2017-33222) to I. Abreu-Sanchez, by the National Science Foundation NSF-MCB 1253444 to L. Vidali, and by the Fulbright Scholar Program to L. Vidali.

*The $\boldsymbol{e}$-Xtra logo stands for "electronic extra" and indicates that one supplementary figure and five supplementary videos are published online.

The author(s) declare no conflict of interest.

() 2020 The American Phytopathological Society respectively) (Albert et al. 2010a). This interaction induces PAMP-triggered or MAMP-triggered immunity (Boller and Felix 2009; Peng et al. 2018). Commonly studied PRR are FLS2 and EFR1 (Altenbach and Robatzek 2007; Kunze et al. 2004), which recognize bacterial proteins, and LYK5, which, together with LYK4, are the primary detectors of fungal chitin (Cao et al. 2014). LYK4/5, upon detection of chitin oligosaccharides, activates CERK1, a major coreceptor involved in chitin response (Miya et al. 2007). Upon PRR activation, cytoplasmic $\mathrm{Ca}^{2+}$ concentration increases, and a simultaneous mitogen-activated protein kinase (MAPK) phosphorylation cascade triggers a defense response that includes synthesis of defense hormones, defense gene activation, and, in vascular plants, lignification of the cell wall (Ponce de León and Montesano 2017).

Bryophytes such as the plant model Physcomitrella patens share characteristics with the common ancestor between bryophytes and vascular plants; this makes $P$. patens a great model to study the evolution of the plant immune response (Alvarez et al. 2016; Lang et al. 2008; Nishiyama et al. 2003). Initially, $P$. patens was used as a model system to study plant immune response because of its sensitivity to both the bacterium Erwinia carotovora and the fungus Botrytis cinerea (Ponce de León et al. 2007). Such infections result in the manifestation of many disease symptoms in moss, including tissue maceration, cytoplasmic shrinkage, chloroplast darkening, and expression of defense-related genes (Ponce de León et al. 2007). Further characterization of $P$. patens immune response triggered by fungi of the genus Pythium showed that moss activates a response common to flowering plants, including accumulation of phenolic compounds and callose in the cell wall, reactive oxygen species (ROS) production, cell death, and accumulation of jasmonic acid and its precursor, 12-oxophytodienoic acid (Oliver et al. 2009). Pythium infection also results in the transcription of defense-related genes such as $P A L, C H S, L O X$, and $P R-1$ (Oliver et al. 2009). In a recent study, Alvarez et al. (2016) performed an in-depth characterization of transcriptional activation in $P$. patens following Pectobacterium carotovorum infection. This study allowed for the identification of pathways involved in defense response against pathogens. Furthermore, in this study, several genes coding for cell wall reinforcement such as dirigent-like proteins (DIR) were identified as overexpressed (Alvarez et al. 2016). DIR proteins participate in plant pathogen defense by mediating the production of the cell wall polymers (Davin and Lewis 2000). To activate all of these responses, plants express PRR on 
their plasma membrane as an initial line of defense (Albert et al. 2010b). Interestingly, it has been shown that Physcomitrella patens expresses the PRR CERK chitin receptor (Bressendorff et al. 2016), while homologs of the Arabidopsis thaliana FLS2 and EFR have not been identified (Ponce de León and Montesano 2017). CERK activation by fungal chitin stimulates a downstream response which, in $P$. patens, has similarities to vascular plants, generating an oxidative burst (Lehtonen et al. 2012); and, more recently, demonstrated that MAPK are rapidly activated via phosphorylation in response to chitin elicitation and fungal infection (Bressendorff et al. 2016).

A hallmark of plant immune response to biotic and abiotic stress is the increase of the cytosolic calcium $\left(\mathrm{Ca}^{2+}\right)$ concentration following infection (Seybold et al. 2014). A. thaliana exhibits an increase in $\mathrm{Ca}^{2+}$ in leaves, roots, and guard cells in response to osmotic stress (Cao et al. 2017; Yuan et al. 2014). A. thaliana also responds to herbivore attack, in a glutamatedependent mechanism that triggers a $\mathrm{Ca}^{2+}$ response (Toyota et al. 2018). Drought and salinity stressors in P. patens cause a burst of $\mathrm{Ca}^{2+}$ waves that originate at the base of the gametophore and propagate throughout the whole plant (Storti et al. 2018). Therefore, we hypothesize that, in response to chitin oligosaccharides, similarly to vascular plants, cytosolic $\mathrm{Ca}^{2+}$ will increase and propagate periodically throughout the plant.

To address our hypothesis, we examined $\mathrm{Ca}^{2+}$ response to chitin oligosaccharides in P. patens. The small size and simple cytology of this plant allowed us to analyze the response at the plant level and at the cell level. To visualize cytosolic $\mathrm{Ca}^{2+}$ levels in response to chitin oligosaccharides, we used a $P$. patens line expressing the $\mathrm{Ca}^{2+}$ sensor GCaMPf6 (Chen et al. 2013; Nakai et al. 2001). This line was also transformed with the fluorescent probe Lifeact-Ruby2 (Wu and Bezanilla 2018), which allows simultaneous visualization of the actin cytoskeleton and $\mathrm{Ca}^{2+}$. Our results show that, following exposure to chitin oligosaccharides, $\mathrm{Ca}^{2+}$ oscillation propagates across the whole plant in a dose-dependent manner. At the cellular level, chitin oligosaccharides cause dissipation of the tip-localized actin cytoskeleton essential for cell growth. Additionally, we showed that $\mathrm{Ca}^{2+}$ influx itself (mediated by ionomycin ionophore) is enough to trigger a pathogenesis-like response in $P$. patens.

\section{RESULTS}

\section{Chitin oligosaccharides affect plant growth and morphology in a dose-dependent manner.}

To investigate the effect of chitin oligosaccharides on plant growth and morphology, we performed a growth assay in the presence of increasing concentrations of chitin oligosaccharides. After regenerating $P$. patens protoplasts for 4 days, plants were transferred to growth media containing different concentrations of chitin oligosaccharides. After 3 days of exposure to the chitin oligosaccharides, plants were imaged. Our results show that plant size and morphology are affected by chitin oligosaccharides in a dose-dependent manner (Fig. 1A and B). Interestingly, plants exposed to chitin oligosaccharides have a nonuniform distribution of cellulose on their walls: the calcofluor-white fails to stain the entire plant cell wall. For this reason, for visualization and analysis purposes, we merged the calcofluor-white and chlorophyll autofluorescence channels (discussed below). To quantify the observed morphological changes, we measured plant area and solidity as previously described (Vidali et al. 2007) (Fig. 1B). Plant area and solidity were estimated from the merged calcofluor-white and chlorophyll signals; plant solidity was calculated by computing the ratio of the area over the convex hull area (Vidali et al. 2007). Plant area progressively decreased when plants were exposed to increasing chitin oligosaccharides concentrations; curve fitting using a variable slope model (GraphPad software) produced a half-maximal inhibitory concentration $\left(\mathrm{IC}_{50}\right)$ value of $266 \pm 44 \mu \mathrm{g} / \mathrm{ml}$ (standard error of the mean [SEM]). Plant solidity, which is an indirect estimate of polarized cell growth, progressively increased with higher concentrations of chitin oligosaccharides, assuming values closer to 1, typical of stunted and less-branching plants. For plant solidity values, curve fitting using a variable slope model generated an $\mathrm{IC}_{50}$ value of $373 \pm 126 \mu \mathrm{g} / \mathrm{ml}$ (SEM). To our surprise, we observed that $P$. patens plants showed a nonhomogeneous response to chitin oligosaccharides. Although the majority of plants shared the same altered phenotype, some exhibited a less dramatic phenotype and appeared to be less affected by chitin oligosaccharides; some examples of this are visible in Figure 1A (arrows).

\section{$\mathrm{Ca}^{2+}$ oscillations originate following elicitation with chitin oligosaccharides.}

To examine the effect of $\mathrm{Ca}^{2+}$ at the plant level, we used a $P$. patens line expressing the cytoplasmic $\mathrm{Ca}^{2+}$ sensor GCaMP6f (Chen et al. 2013). We generated single 1-week-old plants, treated them with chitin oligosaccharides, and recorded changes in intracellular $\mathrm{Ca}^{2+}$ levels using the fluorescence microscope. Upon chitin oligosaccharides addition, $\mathrm{Ca}^{2+}$ oscillations propagated throughout the whole plant (Supplementary Fig. S1). Because the chitin oligosaccharides used were a mixture of oligosaccharides with different numbers of $\mathrm{N}$ acetyl-glucosamine units, we sought to investigate whether a well-defined number of units is needed to trigger a response. Hence, we repeated the experiment by using hexa-Nacetylchitohexaose (chitohexaose) and $\mathrm{N}, \mathrm{N}^{\prime}, \mathrm{N}^{\prime \prime}$-triacetylchitotriose (chitotriose) (Fig. 2). We found that chitohexaose triggers a global $\mathrm{Ca}^{2+}$ response equivalent to that of chitin oligosaccharides. These results indicate that a critical number, higher than three $\mathrm{N}$-acetyl-glucosamine units, is required to cause a response in moss (Fig. 2). Similarly to the chitin oligosaccharide treatment, upon chitohexaose application, $\mathrm{Ca}^{2+}$ oscillations propagated throughout the whole plant, with what sometimes appeared to be waves traveling within and between cells (Fig. 2A; compare Supplementary Videos S1, S2, and S3). To quantify the magnitude of the $\mathrm{Ca}^{2+}$ response, we calculated the time averaged of the mean gray values across the time series (30 $\mathrm{min}$ ) for the different chitohexaose and chitotriose concentrations. Our results show that the cytoplasmic $\mathrm{Ca}^{2+}$ increase was dose dependent: as the chitohexaose concentration increased, the time-averaged mean gray values of $\mathrm{Ca}^{2+}$ fluorescence signal also increased (Fig. 2B, left). A similar dose response was observed with the chitin oligosaccharide treatment (Supplementary Fig. S1), whereas chitotriose only triggered a minimal response at the concentrations used (Fig. 2B, left). Curve fitting using the agonist concentration versus response with a three-parameter model generated an effective concentration that provides $50 \%$ of the maximal response $\left(\mathrm{EC}_{50}\right)=2.2 \pm 0.5 \mu \mathrm{M}( \pm \mathrm{SEM})$ for the chitohexaose and an $\mathrm{EC}_{50}=468.3 \pm 189.3 \mu \mathrm{M}$ for the chitotriose. Similarly, the standard deviation of the time-averaged mean gray value increased in treated plants, with an $\mathrm{EC}_{50}=0.9 \pm 0.4 \mu \mathrm{M}$ for the chitohexaose treatment and an $\mathrm{EC}_{50}=101.1 \pm 31.6 \mu \mathrm{M}$ for the chitotriose treatment. This increase in standard deviation indicates that there is a greater amplitude of the $\mathrm{Ca}^{2+}$ peaks when plants are treated with higher concentrations of chitohexaose and, to a much lesser extent, chitotriose (Fig. 2B, right).

The period of the $\mathrm{Ca}^{2+}$ oscillations appeared to be more frequent when the plants were exposed to the higher concentration of chitin oligosaccharides and chitohexaose (Supplementary Fig. S1; Fig. 2A).To determine the changes in frequency, we used a peak detection algorithm (discussed below). This algorithm 
analyzes the fluorescence intensity values across all of the pixels in a plant over time (Fig. 2C), and detects the $\mathrm{Ca}^{2+}$ peaks in a group of nine pixels across the time series (Fig. 2D). The period of the oscillation in a plant is calculated from the average time elapsed between peaks (Fig. 2D) for all of the pixels, and the peak frequency is computed from the period. When plants were exposed to an increasing concentration of chitin oligosaccharides or chitohexaose, the number of detected $\mathrm{Ca}^{2+}$ peaks increased
A

Control

Chitin

$160 \mu \mathrm{g} / \mathrm{ml}$

Chitin

$260 \mu \mathrm{g} / \mathrm{ml}$

Chitin

$380 \mu \mathrm{g} / \mathrm{ml}$

Chitin

$590 \mu \mathrm{g} / \mathrm{ml}$

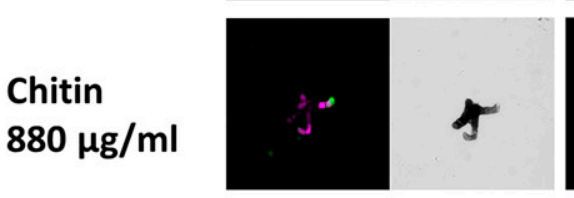

Chitin

$1300 \mu \mathrm{g} / \mathrm{ml}$
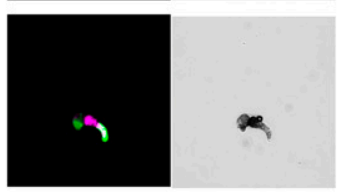

Chitin

$2000 \mu \mathrm{g} / \mathrm{ml}$

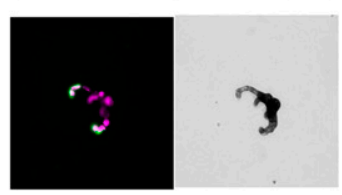

B

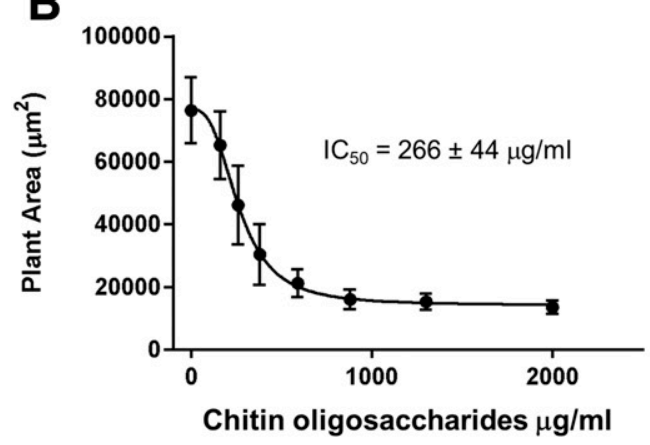

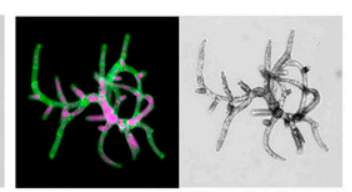
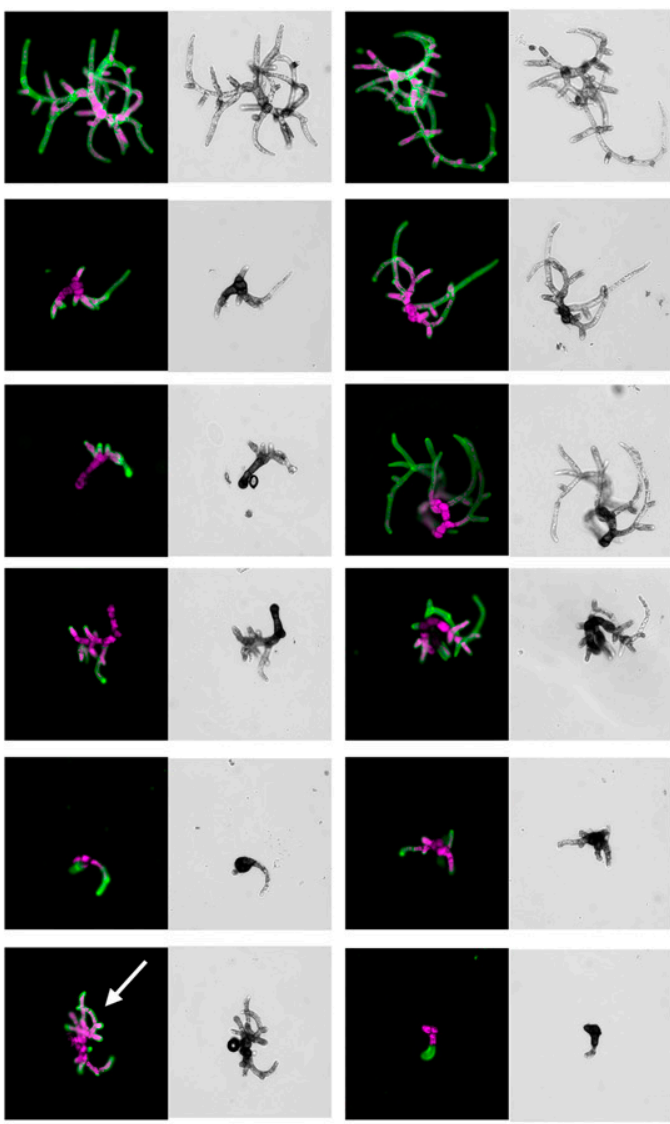

Y
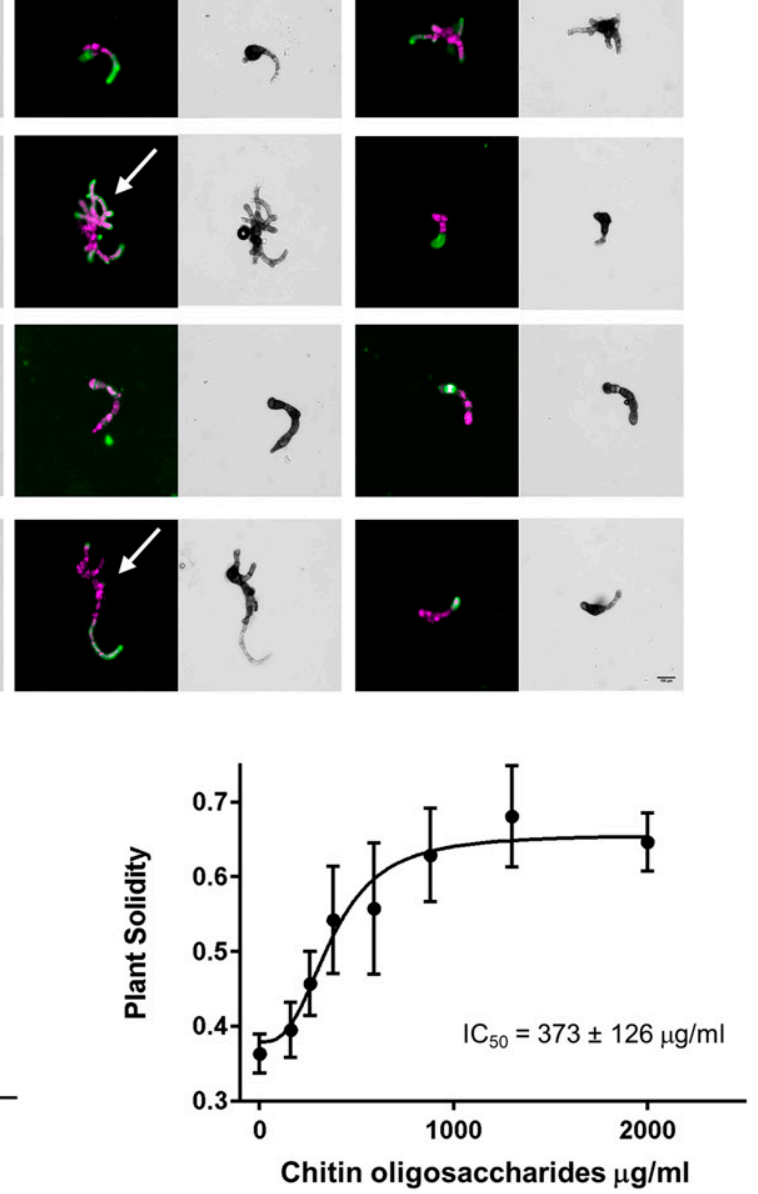

Fig. 1. Exposure to chitin oligosaccharides affects plant size and morphology in a dose-dependent manner. A, Representative images of 1-week-old plants acquired via epifluorescence microscopy. In the panel, green represents calcofluor staining and magenta the chlorophyll autofluorescence. Arrows indicate examples of plants exhibiting a less severe phenotype in response to chitin oligosaccharides. B, Exposure to increasing concentrations of chitin oligosaccharides results in a reduction of plant area and an increase in plant solidity. Quantification of plant area and solidity in plants exposed to increasing concentration of chitin oligosaccharides. Number of plants analyzed: control plants $=75$, plants exposed at $160 \mu \mathrm{g} / \mathrm{ml}=74, \mathrm{plants}$ exposed at $260 \mu \mathrm{g} / \mathrm{ml}=79$, plants exposed at $380 \mu \mathrm{g} / \mathrm{ml}=75$, plants exposed at $590 \mu \mathrm{g} / \mathrm{ml}=78$, plants exposed at $880 \mu \mathrm{g} / \mathrm{ml}=76$, plants exposed at $1,300 \mu \mathrm{g} / \mathrm{ml}=78$, and plants exposed at $2,000 \mu \mathrm{g} / \mathrm{ml}=75$. Three biological replicates were performed. $\mathrm{IC}_{50}=$ half-maximal inhibitory concentration. 
A

Chico-

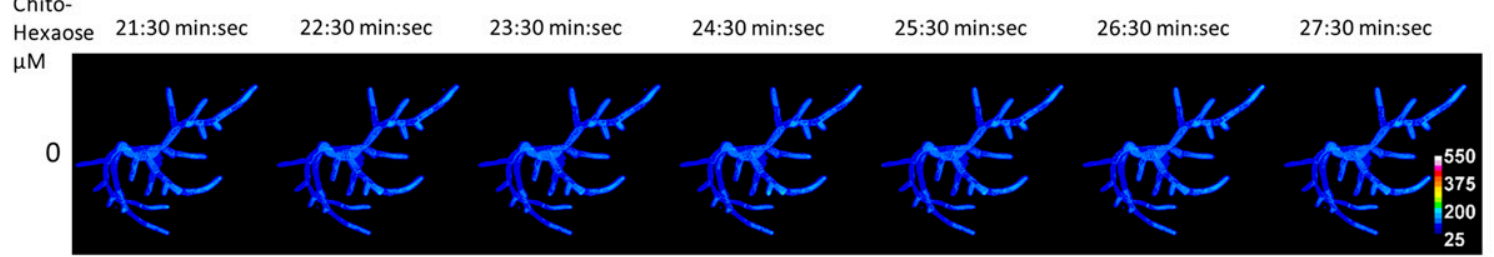

1

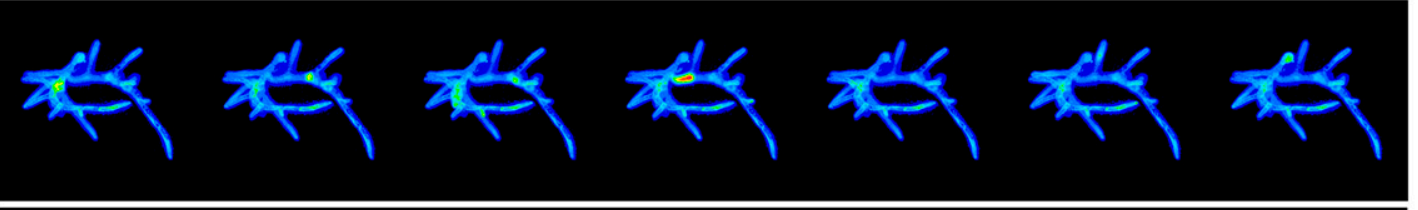

100
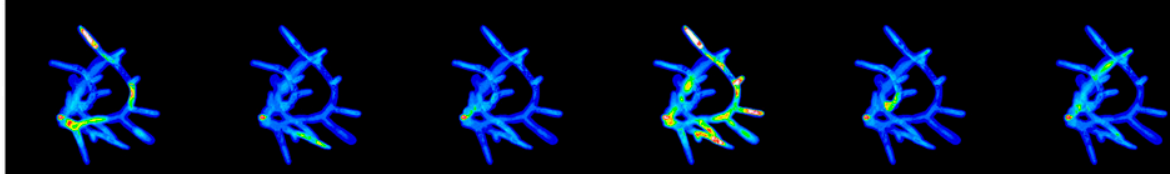

B
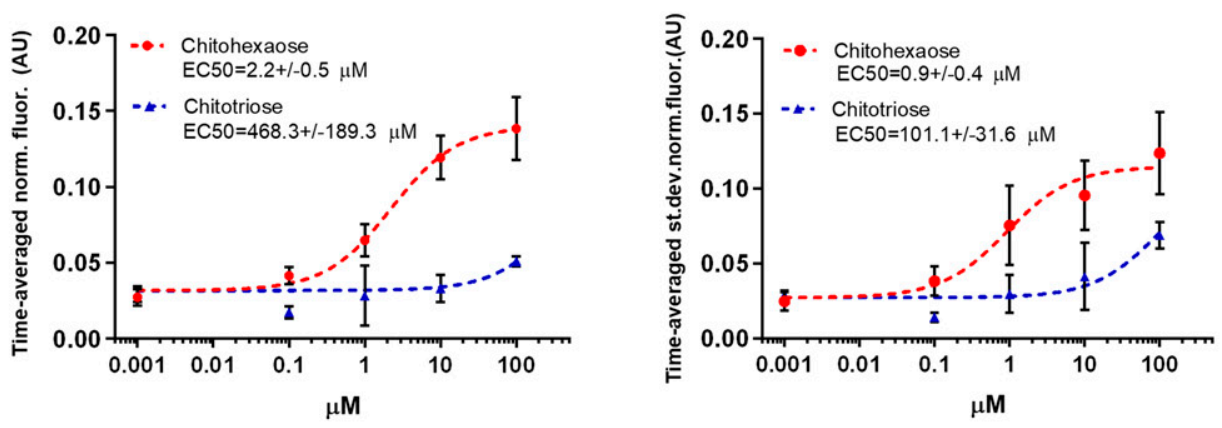

C
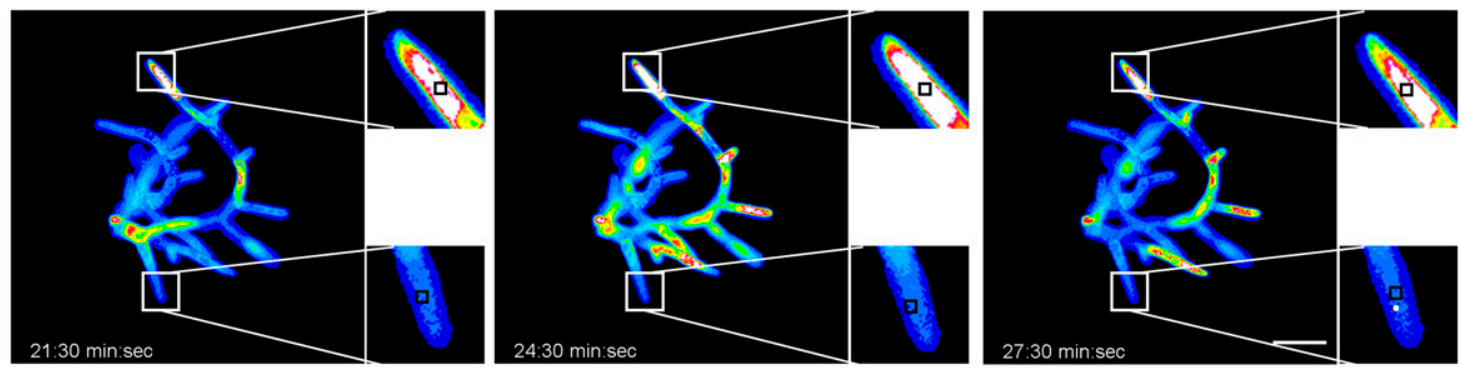

D

E
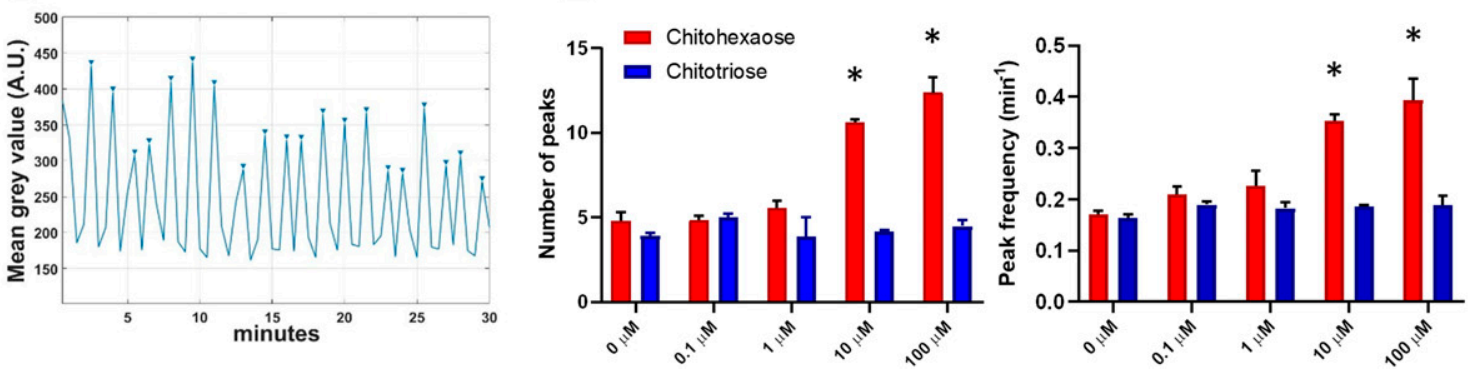

Fig. 2. Chitohexaose affects cytosolic $\mathrm{Ca}^{2+}$ concentrations. A, Plants at concentrations of $0,1,0.1$, and 10 and $100 \mu \mathrm{M}$ chitohexaose. Scale bar $=50 \mu \mathrm{m}$. B, Time-averaged mean gray value (left panel) and the standard deviation (st.dev) (right panel) of the time-averaged mean gray value are normalized by ( $\mathrm{F}-$ $\left.\mathrm{F}_{0}\right) / \mathrm{F}_{0}$ for chitohexaose- and chitotriose-treated plants. Each point represents the average of at least three plants across 30 min of imaging; in total, three independent experiments were performed for each condition. $\mathrm{EC}_{50}=$ effective concentration that provides $50 \%$ of the maximal response. $\mathbf{C}$, Example of a plant treated with $100 \mu \mathrm{M}$ chitohexaose, imaged for $30 \mathrm{~min}$. Side images are examples of the image processing. Each window contains a 3-by-3-pixel grid, highlighted in black (not to scale in the picture). The mean gray value in each grid is measured across time. Each plant results in approximately 200,000 pixel grids. The period of each grid was extracted using a peak detection program in MATLAB. Scale bar $=50 \mu \mathrm{m}$. D, Example of a peak graph for a plant treated with $100 \mu \mathrm{M}$ chitohexaose; the detected peaks are indicated with arrows. E, Number of peaks per plant (left panel) and frequency of peaks in minutes ${ }^{-1}$ (right panel) in plants treated with chitohexaose and chitotriose. In total, three plants were analyzed per treatment per experiment, and three independent experiments were conducted (each treatment had three control plants -no chitin oligosaccharides added). For the chitohexaose-treated plants, the number of peaks and the peak frequency become significantly different from the control at a concentration of $10 \mu \mathrm{M}$ or above. Asterisks indicate a significant difference from the control (adjusted $P$ value $<0.001$ ) (ordinary one-way analysis of variance). For the chitotriose-treated plants, there is no statistically significant difference.

914 / Molecular Plant-Microbe Interactions 
(Fig. 2E; Supplementary Fig. S1). On the other hand, treatment with chitotriose did not significantly increase the number of peaks compared with the control (Fig. 2E). Control plants or plants treated with a low concentration of chitohexaose had very few peaks (Fig. 2E, left). As the chitohexaose concentration increased, the number of peaks that could be detected increased, with a peak frequency of $0.39 \pm 0.04 \mathrm{~min}^{-1}( \pm \mathrm{SEM})$ for the 100 $\mu \mathrm{M}$ chitohexose treatment (Fig. 2E). This frequency corresponds to an average period between peaks of $2.6 \pm 0.3 \mathrm{~min}$. The number of peaks and the peak frequency became significantly different from the control (adjusted $P$ value $<0.001$ ) at a concentration of $10 \mu \mathrm{M}$ or above. Unlike chitohexaose, chitotriose treatment did not affect the number of peaks or the peak frequency (Fig. 2E). A similar result was obtained when plants were treated with chitin oligosaccharides (Supplementary Fig. S1E); when plants were treated with a concentration of $0.2 \mathrm{mg} / \mathrm{ml}$ or above, the number of peaks increased and the average period was approximately 2 min/peak (Supplementary Fig. 1E).

\section{Chitin oligosaccharides dissipate F-actin at the tip of the cell.}

$P$. patens cells have a dynamic cortical actin cytoskeleton and a fluctuating apical accumulation of F-actin filaments focused at the tip of the cell (Furt et al. 2013; Vidali et al. 2009). The latter is essential for growth, as demonstrated by growth inhibition caused by pharmacological inhibition of F-actin (Furt et al. 2013; Vidali et al. 2009) and mathematical modeling (Bibeau et al. 2018). Because $P$. patens plants exposed to chitin oligosaccharides exhibited reduced growth and altered solidity, we decided to investigate whether the observed growth phenotype is due to chitin-oligosaccharide-induced defects in the F-actin cytoskeleton of filamentous tip growing cells. We generated a line that expresses the Lifeact-Ruby 2 fluorescent probe in our cytoplasmic $\mathrm{Ca}^{2+}$ sensor GCaMP6f cell line, which allows visualization of the actin cytoskeleton. We imaged the actin cytoskeleton following treatment with chitin oligosaccharides at $100 \mu \mathrm{g} / \mathrm{mg}$. Interestingly, the F-actin-enriched spot at the tip of the cell dissipated within seconds after chitin oligosaccharide exposure (Fig. 3A; Supplementary Videos S4 and $\mathrm{S} 5$ ), and the cell stopped growing. This result shows that the Factin cytoskeleton is affected by chitin oligosaccharides in a fast way. Furthermore, following the addition of chitin oligosaccharides, the $\mathrm{Ca}^{2+}$ oscillation pattern at the tip region appeared altered. In untreated cells, the oscillations were periodic and restricted to the apical domain, exhibiting frequent peaks of variable amplitude. After application of chitin oligosaccharides, the $\mathrm{Ca}^{2+}$ signal not only was restricted to the apical domain but also was observed throughout the whole cell, and fewer $\mathrm{Ca}^{2+}$ peaks were observed when analyzing the apical region of the tip cell (Fig. 3B, compare top and bottom).

\section{Chitin oligosaccharides and the calcium-ionophore ionomycin induce expression of defense-related genes.}

To test whether $\mathrm{Ca}^{2+}$ influx had a biological significance in the pathogenesis response of $P$. patens, we analyzed the expression of the pathogenesis-responsive genes phenylalanine ammonia lyase 4 (PpPAL4), chalcone synthase (PpCHS), and PpCERK1 (homolog of the chitin elicitor receptor kinase 1 from Arabidopsis) (Bressendorff et al. 2016; Oliver et al. 2009) in plants treated with chitin oligosaccharides or the $\mathrm{Ca}^{2+}$ ionophore ionomycin (Fig. 4). As previously shown, chitin oligosaccharides induce expression of PpPALA, PpCHS, and PpCERKI in a gradual manner, with the maximum expression observed at $60 \mathrm{~min}$. Ionomycin treatment also induces the expression of pathogenesis-responsive genes, although there is no obvious gradual response, proving that the gene expression is $\mathrm{Ca}^{2+} \mathrm{de}-$ pendent. The intensity of the transcriptional response triggered by ionomycin is generally lower than with chitin oligosaccharides, except at short exposure times, when relative expressions of PpCHS and PpCERKl were similar in both treatments.

\section{DISCUSSION}

The main findings of this study are that $P$. patens treated with chitin oligosaccharides exhibits $\mathrm{Ca}^{2+}$ oscillations and that the $\mathrm{Ca}^{2+}$ oscillations affect plant growth via the depolymerization of the cytoskeleton at the cell tip. These conclusions were based on the following experimental evidence. First, we demonstrated that chitin oligosaccharides affect plant growth and morphology in a dose-dependent manner. Next, we observed and characterized the $\mathrm{Ca}^{2+}$ oscillations that originated following elicitation with chitin oligosaccharides. We then provided evidence that chitin oligosaccharides dissipate F-actin at the tip of the cell, important for cell growth. Finally, we reported that several defense-related genes that are expressed in chitinoligosaccharide-treated plants can be induced by artificially increasing cytosolic $\mathrm{Ca}^{2+}$. Together, these results strongly support the hypothesis that $P$. patens immune response triggered by chitin oligosaccharides is $\mathrm{Ca}^{2+}$ mediated.

The increase in cytosolic $\mathrm{Ca}^{2+}$ that we observed in $P$. patens is common to the response of vascular plants to various biotic stresses. Application of chitin oligosaccharides $(100 \mu \mathrm{g} / \mathrm{ml})$ on leaves of 14- to 16-day-old seedlings resulted in oscillation of cytoplasmic $\mathrm{Ca}^{2+}$ that lasted approximately $20 \mathrm{~min}$ (Keinath et al. 2015). In the same study, it was reported that $100 \mathrm{nM}$ bacterial-derived peptide flagellin caused a systemic $\mathrm{Ca}^{2+}$ increase that lasted for $30 \mathrm{~min}$ (Keinath et al. 2015). Ma et al. (2017) also detected intracellular $\mathrm{Ca}^{2+}$ stimulation induced by flagellin as well as by the pathogen Pseudomonas syringae. Interestingly, our dose-response results showed an $\mathrm{EC}_{50}$ for the $\mathrm{Ca}^{2+}$ response to the chitohexaose of 0.9 to $2.2 \mu \mathrm{M}$, which was very similar to the binding affinity of the A. thaliana chitin receptor AtLYK5 for chitooctaose $(\mathrm{Kd}=1.72 \mu \mathrm{M})(\mathrm{Cao}$ et al. 2014), which suggests that a similar type of receptor may mediate the response in Physcomitrella patens. Furthermore, we observed that six but not three $\mathrm{N}$-acetyl-glucosamine units are required to trigger a $\mathrm{Ca}^{2+}$ response-unlike chitohexaose, chitotriose did not show a significant response. The precise minimum number of units required will need further investigation but, in A. thaliana, Cao et al. (2014) showed that chitohexaose but not chitopentaose triggered an immune response via an increase in ROS. In our work, the transcript levels of three defense-related genes (PAL4, CHS, and CERK1) increased in chitin-oligosaccharide-treated plants and in plants treated with a $\mathrm{Ca}^{2+}$ ionophore. The same transcripts were found to be increased following pathogen attack in P. patens (Alvarez et al. 2016; Bressendorff et al. 2016; Oliver et al. 2009; Ponce de León et al. 2007), suggesting that a link exists between $\mathrm{Ca}^{2+}$ signaling and the moss's response to pathogens.

The actin cytoskeleton is involved in pathogen response as a defense mechanism against microbe penetration (Hardham et al. 2007; Schmidt and Panstruga 2007). In vascular plants, the actin cytoskeleton network thickens at the site of infection, and its pharmacological inhibition facilitates infections (Higaki et al. 2011). Henty-Ridilla et al. (2013) showed that, in epidermal cotyledon cells, treatment with $1 \mu \mathrm{M}$ chitin increased actin filament abundance. These results are in discordance with what we observed in apical cells. However, our study focused specifically on tip growing cells. Hence, it would be interesting to investigate whether the observed modulation in the A. thaliana cytoskeleton by chitin was also observed in A. thaliana tip growing cells (root hairs and pollen tubes).

In $P$. patens plants subjected to abiotic stresses such as $\mathrm{NaCl}$, sorbitol, and mannitol and upon osmotic changes, cytosolic 
$\mathrm{Ca}^{2+}$ concentration rises in a systemic way (Storti et al. 2018). Together with our results, the study by Storti et al. (2018) provides evidence that different kinds of stresses, detected by different receptors, have a common pathway that converges in triggering a $\mathrm{Ca}^{2+}$ increase. However, how plants discriminate between different types of stresses is still unknown. Importantly, by identifying an additional $\mathrm{Ca}^{2+}$-dependent pathway, our study gets us closer to understanding the mechanisms

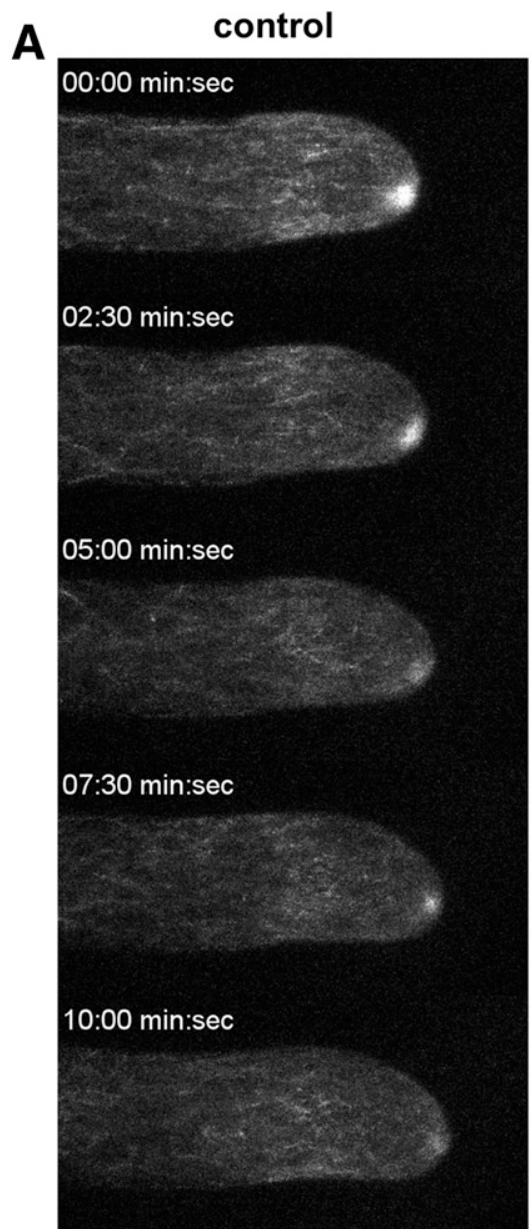

\section{+ chitin oligosaccharides}

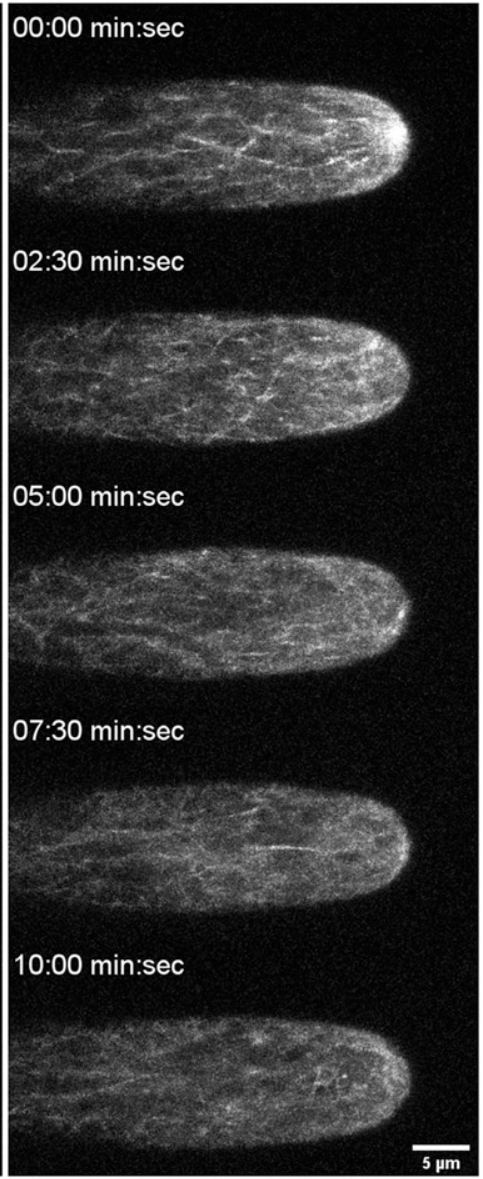

B

Control - apical $\mathrm{Ca}^{2+}$ signal

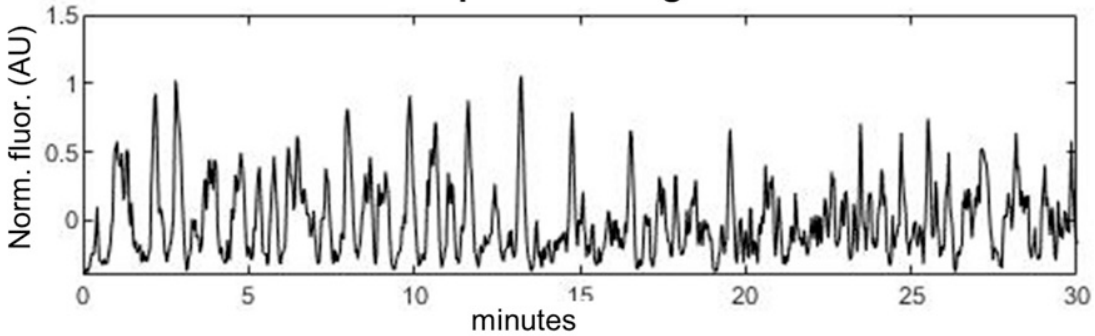

Chitin oligosaccharides treated - apical $\mathrm{Ca}^{2+}$ signal

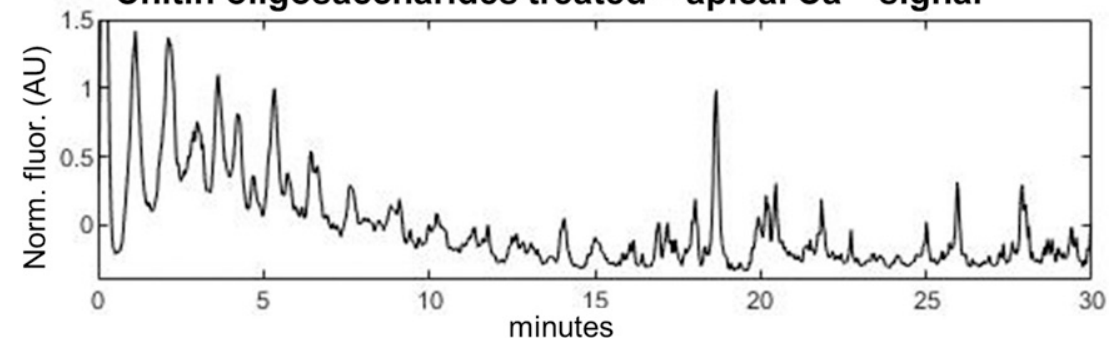

Fig. 3. Chitin oligosaccharides treatment dissipates the apical F-actin focus and alters $\mathrm{Ca}^{2+}$ oscillations in tip cells. A, Chitin oligosaccharides (100 $\left.\mu \mathrm{g} / \mathrm{ml}\right)$ dissipate the actin spot at the tip of the cell. Z-projections of five optical slices acquired with confocal laser scanning microscopy. Actin is labeled with LifeactRuby2. B, Chitin oligosaccharides oscillation at the tip is affected by chitin oligosaccharides. Graphs show representative traces for the Ca ${ }^{2+}$ concentration of control and chitin-oligosaccharide-treated cells. The $\mathrm{Ca}^{2+}$ fluorescence was analyzed using the MATLAB program that analyzed pixel values above zero for every frame; the pixels' gray values were averaged and normalized by subtracting the average of the time series, and dividing the result by the standard deviation of the time series. Number of plants analyzed: five control plants and four plants exposed at $100 \mu \mathrm{g} / \mathrm{ml}$. Three independent experiments were performed. 
behind the capacity of the plant to respond differently to different stresses.

A. thaliana guard cells and root hairs show a different $\mathrm{Ca}^{2+}$ response to flagellin and chitin oligosaccharides than to epidermal cells, indicating that the response is tissue specific (Keinath et al. 2015). Interestingly, when the effect of osmotic stress was investigated in a $P$. patens 1- to 2-month-old leafy gametophore, the response at the base of the gametophore was four times higher than in the phyllids (Storti et al. 2018). Hence, future studies will be needed to investigate how different $P$. patens cell types at different developmental stages respond to chitin oligosaccharides. We focused on the 1-week-old $P$. patens plant because it can be imaged in the field of view of the microscope at low magnification, capturing the response of the organism in its entirety. Our investigation was limited to the protonemal tissue, which is mainly composed of chloronemata cells and of only a limited amount of caulonemata cells. Future experiments should focus on studying the difference in response to chitin between caulonemata and chloronemata, which could be achieved with an experimental setting similar to what we used here to analyze $\mathrm{Ca}^{2+}$ at the single-cell level (confocal microscopy).

Remarkably, we observed two different types of oscillatory behavior. Growing protonemata showed an oscillatory tipfocused $\mathrm{Ca}^{2+}$ gradient (Bascom et al. 2018), similar to the one observed in other plant tip growing cells (Monshausen et al. 2008; Pierson et al. 1996), whereas chitin-oligosaccharideinduced oscillations were not tip focused, and appeared to behave in a wave-like fashion, sometimes encompassing the whole plant. Furthermore, the period of the tip-focused oscillations was faster, with only approximately 30 s between peaks, while the period of the chitin oligosaccharides-induced oscillations is slower, with approximately $2 \mathrm{~min}$. elapsing between peaks. Future studies addressing the identity, location, and activity of $\mathrm{Ca}^{2+}$ transporters (channels and pumps) should help clarify how these two different oscillatory behaviors are generated and maintained.

Our study suggests that chitin-oligosaccharide-triggered immune response in moss is $\mathrm{Ca}^{2+}$ dependent. This response has been previously reported in vascular plants, suggesting that the immune response among land plants could be conserved. Future research is needed to identify the chitin receptors in moss and to uncover the molecular mechanism between the $\mathrm{Ca}^{2+}$ and immune responses. Published evidence shows that the $P$. patens genome harbors four homologs of the A. thaliana chitin receptor AtCERK1 (Bressendorff et al. 2016). Furthermore, $P$. patens encodes at least two copies of the LysM receptor kinases (Zhang et al. 2009), which are essential for chitin recognition in A. thaliana (Cao et al. 2014), and at least three copies of genes encoding homologs of the LYP receptor-like proteins similar to OsCEBiP (Zhang et al. 2009). Identifying whether the $P$. patens receptors mediate the observed $\mathrm{Ca}^{2+}$ response would show whether this response is conserved between mosses and vascular plants. Our study on land plant immune response sets the basis for future characterization of $\mathrm{Ca}^{2+}$ signaling of nonvascular land plant-microbe interactions.

\section{MATERIALS AND METHODS}

Biological material and growth conditions.

All cell lines used in this study were derived from the moss Physcomitrella patens (Hedw.) Bruch \& Schimp., Gransden strain. $P$. patens cell lines were cultured and transformed using established methods (Liu and Vidali 2011).

\section{Morphometric analysis.}

$P$. patens protoplasts were regenerated for 4 days in medium containing $10 \mathrm{mM} \mathrm{CaCl}$ and $6 \%$ mannitol; after regeneration (4 days), protoplasts were transferred to growth medium containing increasing concentrations $(160,260,380,590,880$, 1,300 , and $2,000 \mu \mathrm{g} / \mathrm{ml}$ ) of chitin oligosaccharides (Tokyo Chemical Industry-TCI). Three days after exposure to chitin oligosaccharides, plants were stained with a working concentration of calcofluor-white (dissolved in water) at $10 \mu \mathrm{g} / \mathrm{ml}$ to mark the outline of the plants. Plants were imaged with an inverted epifluorescence microscope Axiovert 200M (Zeiss). The calcofluor-white signal was imaged with a 4',6-diamidino2-phenylindole filter, and the chlorophyll auto fluorescence was detected by using the filter cube GFP-30-LP-B-zhe zero (Semrock Brightline). Because the chitin-oligosaccharidetreated plants exhibited nonuniform calcofluor-white staining, for analysis purposes, we merged the calcofluor-white and chlorophyll autofluorescence channel. Plant area and solidity were measured by using an ImageJ Morphology macro, as previously described (Galotto et al. 2019).

\section{Generation of a moss line expressing the $\mathrm{Ca}^{2+}$ sensor GCaMP6f and Lifeact-Ruby2.}

To construct the plasmid for expression of GCaMP6f in P. patens, we amplified the GCaMP6f open reading frame from
PpCHS

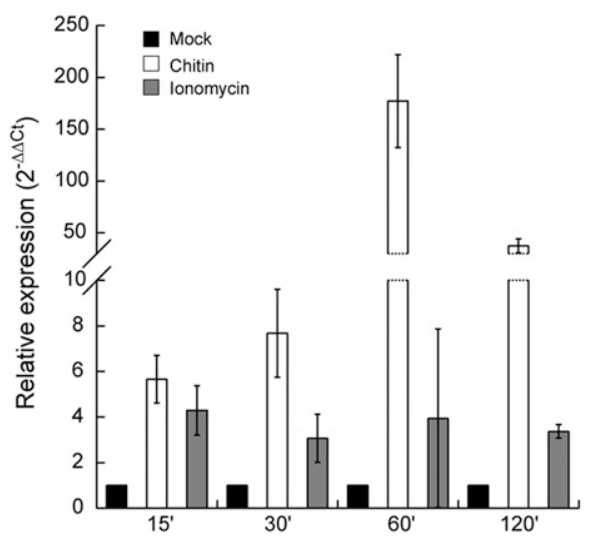

PpPAL4

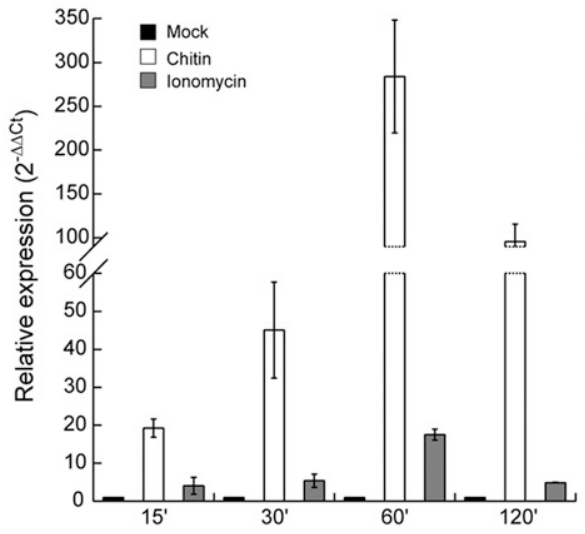

PPCERK1

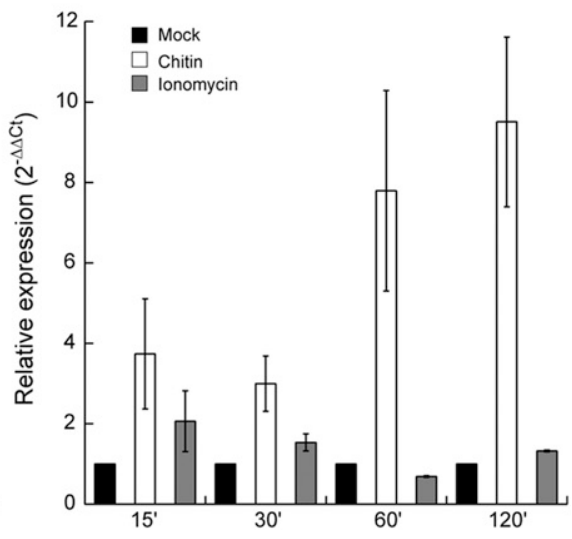

Fig. 4. Expression of pathogenesis-related genes in response to chitin oligosaccharides and ionomycin treatment. Real-time reverse-transcription PCR of $C H S$, $P A L 4$, and CERK1 transcript levels as fold change relative to the wild-type mock treatment at times specified after chitin oligosaccharides (100 $\mu \mathrm{g} / \mathrm{ml})$ or ionomycin $(100 \mathrm{mM})$ treatment. Data points represent an average of two to four independent experiments, each with two technical replicates for each PCR. Error bars represent standard error of the mean. 
plasmid pGP-CMV-GCaMP6f (Chen et al. 2013) (AddGene) with forward and reverse primers containing attB1 and attB2, respectively. The PCR fragment was Gateway cloned into the pDONR207 vector via BP clonase (Thermo) and the product transferred via LR clonase (Thermo) to pTH-Ubi-Gate, a vector with the Gateway cloning site downstream of the maize ubiquitin promoter; this vector provides hygromycin resistance for selection of transformed moss plants (Vidali et al. 2007). Moss protoplasts were simultaneously transformed with the pTHUbi-GCaMP6f construct and a pTZ-Ubi-lifeact-Ruby2 (Vidali et al. 2009; Wu and Bezanilla 2018) construct to label the actin cytoskeleton. Stable transformants were identified by two rounds of selection of hygromycin $(15 \mu \mathrm{g} / \mathrm{ml})$ and zeocin $(50 \mu \mathrm{g} / \mathrm{ml})$.

\section{$\mathrm{Ca}^{2+}$ imaging.}

$P$. patens plants expressing GCaMP6f were imaged with a Zeiss Axio Observer.A1 equipped with a motorized microscope stage. The plants were imaged with a $\times 10$ objective lens (NA $0.25)$. The $\mathrm{Ca}^{2+}$ sensor was excited with a mercury lamp X-Cite series 120 PC EXFO. The microscope slides were prepared with agar pads as previously reported (Galotto et al. 2019). Two microscope slides ( 75 by $25 \mathrm{~mm}$ ), one with chitinoligosaccharide-treated plants and one with untreated plants, were mounted on a 50-by-76-mm slide. Plants were imaged 10 min after the application of chitin oligosaccharide solution. Plants were treated with increasing concentrations of chitin oligosaccharides (Tokyo Chemical Industry-TCI) at 0, 0.002, $0.02,0.2,2$, and $20 \mathrm{mg} / \mathrm{ml}$, and chitohexaose and chitotriose (Cayman Chemical) at $0,0.1,1,10$, and $100 \mu \mathrm{M}$. For imaging, the positions of single plants were saved as spatial coordinates by using the software Micro-Manager (Edelstein et al. 2014). Single plants were then imaged every $30 \mathrm{~s}$ for $30 \mathrm{~min}$. To enhance sensitivity, a binning of 2 was applied to the CCD camera (Cool-SNAP Photometrics).

\section{$\mathrm{Ca}^{2+}$ time-series image analysis.}

The time series of $P$. patens expressing the $\mathrm{Ca}^{2+}$ sensor GCaMP6f exposed to chitin oligosaccharides was analyzed with an ImageJ macro (available upon request). For each frame in the series, the macro applied a thresholding function that masks the background signal; the masked images were used to analyze the $\mathrm{Ca}^{2+}$ peaks in chitin oligosaccharides and chitohexaose- and chitotriose-treated plants (discussed below). In addition, the ImageJ macro recorded the mean gray value of each image. To analyze the chitin-oligosaccharide-treated plants, the collected mean gray values were normalized with an $\mathrm{R}$ script (available upon request) as follows. The $\mathrm{R}$ program computes the average of the mean gray value for each control plant across the time series (control time-series mean), and averages this time-series mean for the three control plants in one data set (F0). The data for each treated plant in the experiment were then normalized in the following way. For each frame in the time series, F0 was subtracted from each mean gray value $(\mathrm{F})$ and then divided by $\mathrm{F} 0$, resulting in $(\mathrm{F}-\mathrm{F} 0) / \mathrm{F} 0$. The resulting values were averaged over time (normalized time-series mean). For chitohexaose and chitotriose, normalization was done by finding the baseline for each individual time series (R package "baseline", function "modpolyfit") and subtracting the baseline from the complete time series, then dividing the resulting values by the baseline $(\mathrm{F}-\mathrm{F} 0) / \mathrm{F} 0$. This approach has the advantage that the water-treated controls can be used for the subsequent curve-fitting analysis. For each experiment from all treatments, the $(\mathrm{F}-\mathrm{F} 0) / \mathrm{F} 0$ value was averaged for the three observed plants. An increase in the above value was an indication of higher $\mathrm{Ca}^{2+}$ levels in the plant but did not provide information about fluctuations in the $\mathrm{Ca}^{2+}$ levels.
To estimate this, we analyzed the standard deviation of the normalized time-series mean, which provided information about the variance of the signal over time. This analysis was repeated for each chitin oligosaccharides concentration used.

\section{$\mathrm{Ca}^{2+}$ peak detection analysis.}

The masked images produced by the ImageJ macro (discussed above) were analyzed using a MATLAB script (available upon request) to identify peaks in the signal that could constitute oscillatory behavior. The program uses a rolling 3-by-3-pixel grid to detect the position occupied by the plant throughout the video. For each frame, the grid scans through the image and records the pixel value of a 3-by-3-pixel area. Only the grids with positive values were considered to be part of a plant. When all of the positive pixels were identified in one plant, the MATLAB script computed the mean of the pixel values at each time point. The script also calculated the standard deviation of the mean for the entire time series. The script then found the $\mathrm{Ca}^{2+}$ peaks corresponding to each pixel in the complete time series. A signal was considered a peak when its intensity was greater than a fraction of the standard deviation $(0.345 \times$ standard deviation) from the mean gray value of the plant over the complete time series. Once the peak detection was completed, the number of peaks and the period between peaks was measured with a built-in MATLAB function (findpeaks). The results from all pixels were averaged to get a value per plant, and the value from three plants was averaged by experiment.

\section{Confocal imaging of actin and $\mathrm{Ca}^{2+}$.}

The $P$. patens plants expressing the $\mathrm{Ca}^{2+}$ sensor GCaMP6f also expressed the fluorescent probe Lifeact-Ruby 2 for simultaneous visualization of $\mathrm{Ca}^{2+}$ and actin cytoskeleton. Plants were cultured in 35-mm glass-bottom dishes in $\mathrm{PpNO}_{3}$ medium, as previously described (Furt et al. 2013). Such plants were imaged with a Leica SP5 scanning confocal microscope. The $\mathrm{Ca}^{2+}$ sensor was excited via the 488-nm argon laser; the Lifeact-Ruby2 fluorophore was excited with the 561-nm laser. Before imaging, plants were treated with $200 \mu \mathrm{l}$ of liquid medium containing chitin oligosaccharides at a final concentration of $100 \mu \mathrm{g} / \mathrm{ml}$. Plants were imaged with $\times 63$ lens (N.A. 1.4), zoom $=5$, five Z-planes separated by approximately $1.5 \mu \mathrm{m}$, every $2 \mathrm{~s}$ for $30 \mathrm{~min}$. F-actin images were Z-projected using maximum intensity, contrast enhanced by normalizing to the stack's histogram, and filtered with a Gaussian blur filter set to a $\sigma=0.5$; all processing was done with ImageJ.

\section{$\mathrm{Ca}^{2+}$ confocal image analysis.}

The $P$. patens plants expressing the $\mathrm{Ca}^{2+}$ sensor GCaMP6f were analyzed with an ImageJ macro (available upon request) to adjust for their growth during analysis. The input for the macro consisted of the coordinates of the tip at the start and end of the time series and a freehand selection of the tip area. The macro created a black mask around the cell boundary that moved at the growth rate of the cell. The output consisted of masked images for the time series of the $\mathrm{Ca}^{2+}$ and F-actin channels. The output images from the previous macro were analyzed for changes in fluorescence intensity with a MATLAB script (available upon request). For every frame, the pixels with values $>0$, which correspond to the $\mathrm{Ca}^{2+}$ signal, were averaged and normalized by subtracting the average of the time series and dividing the result by the standard deviation of the time series. The resulting value was then plotted over time.

RNA extraction and real-time reverse-transcription PCR.

The effect of chitin oligosaccharides and ionomycin in gene expression was tested as follows. P. patens plants grown as indicated were transferred to Petri dishes $(5 \mathrm{~cm}$ in diameter $)$ 
containing $2 \mathrm{ml}$ of liquid media supplied with chitin oligosaccharides $(100 \mu \mathrm{g} / \mathrm{ml})$ (Sigma) or ionomycin $(100 \mu \mathrm{M})$ (Invitrogen). At the indicated times $(15,30,60$, and $120 \mathrm{~min})$, excess media was removed and plants were immediately frozen in liquid nitrogen. RNA was obtained from $P$. patens using TriReagent (Life Technologies), treated with DNase Turbo (Life Technologies) to remove DNA contamination, and cleaned with the RNeasy Mini kit (Qiagen). RNA integrity was confirmed by electrophoresis, and possible DNA contamination was tested by PCR using the RNA samples as templates. cDNA was synthesized from 500 ng of DNA-free RNA using a PrimeScript RT reagent Kit (Takara), supplemented with RNase out (Life Technologies). Expression studies were carried out by real-time reverse-transcription PCR (RT-qPCR) using the StepOne plus thermocycler (Applied Biosystems), with the Power SyBR Green master mix (Applied Biosystems). Primers for the pathogenesisresponsive genes PpPALA, PpCHS, and PpCERKI and the reference gene PpTubulin1 were described elsewhere (Bressendorff et al. 2016). The threshold cycle (Ct) was determined in duplicates from independent experiments $(n=2$ to 4$)$. The relative levels of expression were determined using the $2^{-\Delta \Delta \mathrm{Ct}}$ method, using the mock treatment as the reference condition.

\section{ACKNOWLEDGMENTS}

We thank members of the Vidali and Gonzalez-Guerrero lab for their comments and support.

\section{LITERATURE CITED}

Albert, M., Jehle, A. K., Lipschis, M., Mueller, K., Zeng, Y., and Felix, G. 2010a. Regulation of cell behaviour by plant receptor kinases: Pattern recognition receptors as prototypical models. Eur. J. Cell Biol. 89: 200-207.

Albert, M., Jehle, A. K., Mueller, K., Eisele, C., Lipschis, M., and Felix, G 2010b. Arabidopsis thaliana pattern recognition receptors for bacterial elongation factor Tu and flagellin can be combined to form functional chimeric receptors. J. Biol. Chem. 285:19035-19042.

Altenbach, D., and Robatzek, S. 2007. Pattern recognition receptors: From the cell surface to intracellular dynamics. Mol. Plant-Microbe Interact. 20:1031-1039.

Alvarez, A., Montesano, M., Schmelz, E., and Ponce de León, I. 2016 Activation of shikimate, phenylpropanoid, oxylipins, and auxin pathways in Pectobacterium carotovorum elicitors-treated moss. Front. Plant Sci. 7:328.

Bascom, C. S., Jr., Winship, L. J., and Bezanilla, M. 2018. Simultaneous imaging and functional studies reveal a tight correlation between calcium and actin networks. Proc. Natl. Acad. Sci. U.S.A. 115: E2869-E2878.

Bibeau, J. P., Kingsley, J. L., Furt, F., Tüzel, E., and Vidali, L. 2018. F-Actin mediated focusing of vesicles at the cell tip is essential for polarized growth. Plant Physiol. 176:352-363.

Boller, T., and Felix, G. 2009. A renaissance of elicitors: Perception of microbe-associated molecular patterns and danger signals by patternrecognition receptors. Annu. Rev. Plant Biol. 60:379-406.

Bressendorff, S., Azevedo, R., Kenchappa, C. S., Ponce de León, I., Olsen, J. V., Rasmussen, M. W., Erbs, G., Newman, M. A., Petersen, M., and Mundy, J. 2016. An innate immunity pathway in the moss Physcomitrella patens. Plant Cell 28:1328-1342.

Cao, X. Q., Jiang, Z. H., Yi, Y. Y., Yang, Y., Ke, L. P., Pei, Z. M., and Zhu, S. 2017. Biotic and abiotic stresses activate different $\mathrm{Ca}^{2+}$ permeable channels in Arabidopsis. Front. Plant Sci. 8:83.

Cao, Y., Liang, Y., Tanaka, K., Nguyen, C. T., Jedrzejczak, R. P., Joachimiak, A., and Stacey, G. 2014. The kinase LYK5 is a major chitin receptor in Arabidopsis and forms a chitin-induced complex with related kinase CERK1. eLife 3:e03766.

Chen, T.-W., Wardill, T. J., Sun, Y., Pulver, S. R., Renninger, S. L., Baohan, A., Schreiter, E. R., Kerr, R. A., Orger, M. B., Jayaraman, V., Looger, L. L., Svoboda, K., and Kim, D. S. 2013. Ultrasensitive fluorescent proteins for imaging neuronal activity. Nature 499:295-300.

Davin, L. B., and Lewis, N. G. 2000. Dirigent proteins and dirigent sites explain the mystery of specificity of radical precursor coupling in lignan and lignin biosynthesis. Plant Physiol. 123:453-462.
Edelstein, A. D., Tsuchida, M. A., Amodaj, N., Pinkard, H., Vale, R. D., and Stuurman, N. 2014. Advanced methods of microscope control using $\mu$ Manager software. J. Biol. Methods 1:e10.

Furt, F., Liu, Y. C., Bibeau, J. P., Tüzel, E., and Vidali, L. 2013. Apical myosin XI anticipates F-actin during polarized growth of Physcomitrella patens cells. Plant J. 73:417-428.

Galotto, G., Bibeau, J. P., and Vidali, L. 2019. Automated image acquisition and morphological analysis of cell growth mutants in Physcomitrella patens. Methods Mol. Biol. 1992:307-322.

Hardham, A. R., Jones, D. A., and Takemoto, D. 2007. Cytoskeleton and cell wall function in penetration resistance. Curr. Opin. Plant Biol. 10: 342-348.

Henty-Ridilla, J. L., Shimono, M., Li, J., Chang, J. H., Day, B., and Staiger, C. J. 2013. The plant actin cytoskeleton responds to signals from microbe-associated molecular patterns. PLoS Pathog. 9:e1003290.

Higaki, T., Kurusu, T., Hasezawa, S., and Kuchitsu, K. 2011. Dynamic intracellular reorganization of cytoskeletons and the vacuole in defense responses and hypersensitive cell death in plants. J. Plant Res. 124: 315-324.

Keinath, N. F., Waadt, R., Brugman, R., Schroeder, J. I., Grossmann, G., Schumacher, K., and Krebs, M. 2015. Live cell imaging with R-GECO1 sheds light on flg22- and chitin-induced transient $\left[\mathrm{Ca}^{2+}\right]_{\text {cyt }}$ patterns in Arabidopsis. Mol. Plant 8:1188-1200.

Kunze, G., Zipfel, C., Robatzek, S., Niehaus, K., Boller, T., and Felix, G. 2004. The $\mathrm{N}$ terminus of bacterial elongation factor Tu elicits innate immunity in Arabidopsis plants. Plant Cell 16:3496-3507.

Lang, D., Zimmer, A. D., Rensing, S. A., and Reski, R. 2008. Exploring plant biodiversity: The Physcomitrella genome and beyond. Trends Plant Sci. 13:542-549.

Lehtonen, M. T., Akita, M., Frank, W., Reski, R., and Valkonen, J. P. 2012. Involvement of a class III peroxidase and the mitochondrial protein TSPO in oxidative burst upon treatment of moss plants with a fungal elicitor. Mol. Plant-Microbe Interact. 25:363-371.

Liu, Y. C., and Vidali, L. 2011. Efficient polyethylene glycol (PEG) mediated transformation of the moss Physcomitrella patens. J. Vis. Exp. 50:e2560.

Ma, Y., Zhao, Y., and Berkowitz, G. A. 2017. Intracellular $\mathrm{Ca}^{2+}$ is important for flagellin-triggered defense in Arabidopsis and involves inositol polyphosphate signaling. J. Exp. Bot. 68:3617-3628.

Miya, A., Albert, P., Shinya, T., Desaki, Y., Ichimura, K., Shirasu, K., Narusaka, Y., Kawakami, N., Kaku, H., and Shibuya, N. 2007. CERK1, a LysM receptor kinase, is essential for chitin elicitor signaling in Arabidopsis. Proc. Natl. Acad. Sci. U.S.A. 104:19613-19618.

Monshausen, G. B., Messerli, M. A., and Gilroy, S. 2008. Imaging of the Yellow Cameleon 3.6 indicator reveals that elevations in cytosolic $\mathrm{Ca}^{2+}$ follow oscillating increases in growth in root hairs of Arabidopsis. Plant Physiol. 147:1690-1698.

Nakai, J., Ohkura, M., and Imoto, K. 2001. A high signal-to-noise $\mathrm{Ca}\left({ }^{2+}\right)$ probe composed of a single green fluorescent protein. Nat. Biotechnol. 19:137-141

Nishiyama, T., Fujita, T., Shin-I, T., Seki, M., Nishide, H., Uchiyama, I., Kamiya, A., Carninci, P., Hayashizaki, Y., Shinozaki, K., Kohara, Y., and Hasebe, M. 2003. Comparative genomics of Physcomitrella patens gametophytic transcriptome and Arabidopsis thaliana: Implication for land plant evolution. Proc. Natl. Acad. Sci. U.S.A. 100:8007-8012.

Oliver, J. P., Castro, A., Gaggero, C., Cascón, T., Schmelz, E. A., Castresana, C., and Ponce de León, I. 2009. Pythium infection activates conserved plant defense responses in mosses. Planta 230:569-579.

Peng, Y., van Wersch, R., and Zhang, Y. 2018. Convergent and divergent signaling in PAMP-triggered immunity and effector-triggered immunity Mol. Plant-Microbe Interact. 31:403-409.

Pierson, E. S., Miller, D. D., Callaham, D. A., van Aken, J., Hackett, G., and Hepler, P. K. 1996. Tip-localized calcium entry fluctuates during pollen tube growth. Dev. Biol. 174:160-173.

Ponce de León, I., and Montesano, M. 2017. Adaptation mechanisms in the evolution of moss defenses to microbes. Front. Plant Sci. 8:366.

Ponce de León, I., Oliver, J. P., Castro, A., Gaggero, C., Bentancor, M., and Vidal, S. 2007. Erwinia carotovora elicitors and Botrytis cinerea activate defense responses in Physcomitrella patens. BMC Plant Biol. $7: 52$

Schmidt, S. M., and Panstruga, R. 2007. Cytoskeleton functions in plant-microbe interactions. Physiol. Mol. Plant Pathol. 71:135-148.

Seybold, H., Trempel, F., Ranf, S., Scheel, D., Romeis, T., and Lee, J. 2014. $\mathrm{Ca}^{2+}$ signalling in plant immune response: From pattern recognition receptors to $\mathrm{Ca}^{2+}$ decoding mechanisms. New Phytol. 204:782-790.

Storti, M., Costa, A., Golin, S., Zottini, M., Morosinotto, T., and Alboresi, A. 2018. Systemic calcium wave propagation in Physcomitrella patens Plant Cell Physiol. 59:1377-1384. 
Toyota, M., Spencer, D., Sawai-Toyota, S., Jiaqi, W., Zhang, T., Koo, A. J., Howe, G. A., and Gilroy, S. 2018. Glutamate triggers long-distance, calcium-based plant defense signaling. Science 361:1112-1115.

Vidali, L., Augustine, R. C., Kleinman, K. P., and Bezanilla, M. 2007. Profilin is essential for tip growth in the moss Physcomitrella patens. Plant Cell 19:3705-3722.

Vidali, L., Rounds, C. M., Hepler, P. K., and Bezanilla, M. 2009. LifeactmEGFP reveals a dynamic apical F-actin network in tip growing plant cells. PLoS One 4:e5744.
Wu, S. Z., and Bezanilla, M. 2018. Actin and microtubule cross talk mediates persistent polarized growth. J. Cell Biol. 217:3531-3544.

Yuan, F., Yang, H., Xue, Y., Kong, D., Ye, R., Li, C., Zhang, J., Theprungsirikul, L., Shrift, T., Krichilsky, B., Johnson, D. M., Swift, G. B., He, Y., Siedow, J. N., and Pei, Z. M. 2014. OSCA1 mediates osmotic-stress-evoked $\mathrm{Ca}^{2+}$ increases vital for osmosensing in Arabidopsis. Nature 514:367-371.

Zhang, X. C., Cannon, S. B., and Stacey, G. 2009. Evolutionary genomics of LysM genes in land plants. BMC Evol. Biol. 9:183. 